\title{
Non-Existential Indefinites and Semantic Incorporation of PP Complements
}

\author{
Sela Mador-Haim \\ Yoad Winter \\ Technion \\ Technion/NIAS/Utrecht University
}

\section{Introduction}

Non-existential interpretations of indefinites have greatly influenced the development of formal semantic theory, and most notably so since Carlson's (1977) study of genericity and Heim's (1982) and Kamp's (1981) works on indefinites and anaphora. These works stressed the fact that the syntactic environment in which an indefinite appears may determine to a large extent its quantificational interpretation or binding potential. In this paper we show that prepositional phrases (PPs) are among the syntactic environments that lead to non-existential effects with indefinites. We observe the variability in the quantificational force of indefinites when they appear as PP complements, and account for this new observation by synthesizing ideas from previous accounts of semantic incorporation, spatial prepositions and negative polarity items. In a nutshell, we propose that the English $a$ indefinites can act as predicates, and that such predicates are acceptable as direct arguments of locative prepositions. This is very similar to the process that is assumed in theories of semantic incorporation (McNally 1992, Van Geenhoven 1998, Zimmermann 1993). In this account, the interpretation of predicative indefinites is governed not by quantificational elements within the DP but by other particles in the sentence: in our case, the preposition of which the indefinite DP is an argument. This emphasis on external effects on indefinites is similar to theories of the generic, anaphoric, scopal or intensional semantics of indefinites. In this paper we couple the study of indefinites with a semantic domain that is especially rich and heterogeneous: the spatial semantics of the PP. We employ parts of Zwarts and Winter's (2000) treatment of PPs in vector space semantics, relying especially on their analysis of PP semantic structure and monotonicity properties. As we will show, this monotonicity, or its absence, is a major factor that affects the quantificational behavior of predicative indefinites in PP complements - existential, universal or otherwise.

The paper is structured as follows. Section 2 presents the problem of nonexistential indefinites in PP complements. Section 3 introduces our proposal in informal terms and describes its implications. Section 4 fleshes out some central

The second author is grateful for the generous support of the Netherlands Institute of Advanced Study (NIAS), the Utrecht Institute of Linguistics OTS (Utrecht University) and the Netherlands Organization for Scientific Research (NWO). For discussions and remarks we thank Edit Doron, Danny Fox, Berit Gehrke, Manfred Krifka, Louise McNally, Ede Zimmermann, Joost Zwarts and the participants of SALT 17 and the workshop on "funny indefinites" in Berlin (July 2007). 
formal details of our account, and discusses some of its further implications and open problems.

\section{Quantificational Variability of PP Complements}

Indefinites that appear as PP complements show some systematic variations in their interpretation, which to the best of our knowledge have not been observed before. Consider the following scenario: you are driving in Nevada with a friend when suddenly your car runs out of gas. Now consider the following two sentences that you or your friend may utter in response to the situation: ${ }^{1}$

(1) a. We're close to a gas station.

b. We're far from a gas station.

The two sentences obviously have different implications for the fate of your journey. While sentence (1a) means that some gas station must be close to where you are, (1b) does not mean that some gas station is far away. Rather, sentence (1b) conveys the stronger, unfavorable, proposition that all gas stations are far from your location.

This dependency of the quantificational interpretation of indefinites on the preposition cannot be dismissed as a pragmatic effect. It appears in numerous constructions and circumstances where pragmatics is unlikely to matter. For instance, consider the following variation on the contrast in (1).
a. The house is $\left\{\begin{array}{l}\text { at most } \\ \text { less than }\end{array}\right\} 2 \mathrm{~km}$ from a lake.
b. The house is $\left\{\begin{array}{l}\text { at least } \\ \text { more than }\end{array}\right\} 2 \mathrm{~km}$ from a lake.

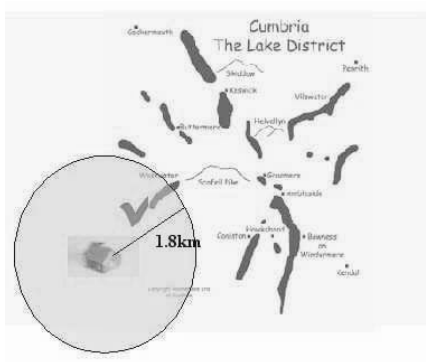

(a)

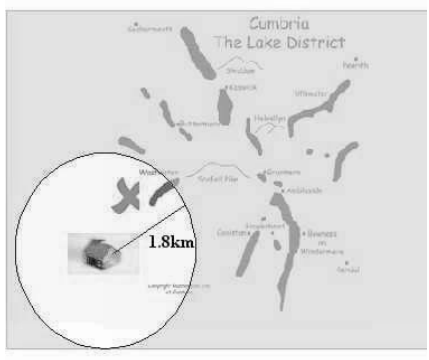

(b)

Figure 1: close tolfar from a lake

Traditionally, we may expect both occurrences of the indefinite a lake in (2) to be interpreted as an existential quantifier. This is indeed the case in (2a), which is

\footnotetext{
${ }^{1}$ We thank Louise McNally for suggesting this example to us.
} 
perfectly acceptable as a description of Figure 1a. The sentences in (2a) are true when there is at least one lake which is less than two kilometers away from the house, as it is the case in Figure 1a. However, as illustrated by the unacceptability of $(2 b)$ in Figure $1 b$, the mere existence of a lake that is more than two kilometers away from the house is not enough to make these sentences true. Apparently, the prominent interpretation of the indefinite in (2b) is universal: these sentences claim that for every lake $x$, the house is at least (more than) $2 \mathrm{~km}$ away from $x$.

The universal interpretation of the indefinites in sentences like (1b) or (2b) is not necessarily their only interpretation. When enough descriptive content is added to it, the indefinite may get a "specific", wide-scope existential interpretation, as would be expected by most theories of indefinites. For instance:

(3) a. We're far from a gas station that I read about in the guide, but I think there must be another one nearby.

b. The house is more than $2 \mathrm{~km}$ from a well-known lake that is very popular among tourists, but there are two smaller lakes in a walking distance of $1 \mathrm{~km}$ which are very nice and much quieter.

In such cases the universal reading of the indefinite may be ruled out. To be sure it is not prominent in the same way it is in cases like (1b) or $(2 b){ }^{2}$ What is crucial for us is that a similar universal/existential discrepancy does not show up in cases like (1a) or (2a). It is quite impossible to think of a context where such sentences would receive a definitely universal interpretation.

Existential/universal variability as in (1) and (2) is also found with the locatives inside and outside. Consider the following examples.

(4) a. The dog is inside a doghouse.

b. The dog is outside a doghouse.

Sentence (4a) is interpreted existentially, as claiming that there is a doghouse $x$ such that the dog is inside $x$. Sentence (4b), on the other hand, is unlikely to be interpreted in this way: its prominent reading entails that the dog is outside all the doghouses.

There are also interpretations of indefinite PP complements that are neither existential nor universal. Consider the following example.

(5) The dog is less than $20 \mathrm{~m}$ outside a doghouse.

Sentence (5) shows a non-existential effect, but one that is not strictly universal. For this sentence to be true the dog should not be inside any doghouse, and there should also be a doghouse $x$ such that the dog is less than 20m from $x$. Thus, in this case the preposition outside contributes the universal import as in (4b) and the measure phrase less than $20 m$ contributes an existential import as in (2a).

Another complex quantificational effect is illustrated in (6) below.

(6) The bird is more than $20 \mathrm{~m}$ above a cloud.

\footnotetext{
${ }^{2}$ Proponents of Schwarzschild's (2002) "singleton indefinites" may prefer to regard the apparently existential indefinites in (3) as universal quantifiers with singleton domains.
} 
Here, by contrast to (5), the measure phrase contributes a universal interpretation but the preposition interferes. In (6), the bird is not claimed to be more than $20 \mathrm{~m}$ above all the clouds. Rather, only clouds that are below the bird are universally quantified over. Clouds above the bird do not matter for assessing the truth of the non-existential interpretation of (6). Intuitively, this semi-universal effect stems from the universal effect triggered by the measure phrase more than $20 \mathrm{~m}$, together with the restriction imposed by the preposition above on the region that is relevant for evaluating (6).

Another way of triggering quantificational effects that are neither existential nor universal is illustrated in the following examples.

a. The house is exactly $100 \mathrm{~m}$ from a lake.

b. The house is between $100 \mathrm{~m}$ and $200 \mathrm{~m}$ from a lake.

While the downward/upward monotonicity of the measure phrases (MPs) in (2) triggers the existential/universal interpretation of the indefinite, the MPs in (7) are non-monotone. ${ }^{3}$ These MPs are equivalent to the following conjunctions of downward monotonic MPs with upward monotonic MPs (e.g. exactly $100 \mathrm{~m}=$ at least $100 \mathrm{~m}$ and at most $100 \mathrm{~m}$ ). Expectedly, the quantificational force given to the indefinites in (7) is semi-existential/semi-universal. Sentence (7a) is equivalent to the claim that the house is at most $100 \mathrm{~m}$ from a lake and at least $100 \mathrm{~m}$ from a lake. Thus, (7a) claims that there is a lake $x$ such that the house is at most $100 \mathrm{~m}$ from $x$ (existential import of at most), and for every lake $y$ the house is at least $100 \mathrm{~m}$ from $y$ (universal import of at least). In other words: the closest lake to the house is exactly $100 \mathrm{~m}$ away from it. The effect in (7b) is similar.

So far we have considered only indefinites with the article $a$ as showing non-existential effects in PP complements. It is important to note that the some indefinites in English do not show similar quantificational variability effects. Consider for instance the following examples, in contrast with sentences (1b) and (4b) above.

(8) We're far from some gas station.

(9) The dog is outside some doghouse.

These examples, in contrast to their parallels with the article $a$, only show an existential interpretation of the indefinite. In the account that we present in the following section, we take this as an important clue for the origins of the non-existential effects with $a$ indefinites.

Moving on to other DPs, we note that similar effects to the ones we observed with $a$ indefinites, also appear with bare plurals and plural definites, and even with singular definites that denote objects with a mereological structure. Consider the following examples.

(10) We're close to/far from lakes.

(11) We're close to/far from the lakes.

\footnotetext{
${ }^{3}$ In Section 4 we say more on the monotonicity properties of MPs and prepositions, and their formal effects on the interpretation of indefinite PP complements.
} 
(12) We're close to/far from the lake.

In (10) and (11), existential/universal quantification over lakes is triggered by the locative expression, in a similar way to the contrast in (1). The statements made in (12) are that we are close to some part of the lake and far from all parts of the lake, respectively. We believe that the variability effects in (10)-(12) stem from the same principles about the interpretation of spatial expressions that we propose in this paper. However, we do not concentrate here on the interpretative strategies in such examples, and leave the semantics of plurals and part-whole structures beyond the scope of this paper.

\section{Incorporation as a Source of Quantificational Variability}

To account for non-existential interpretations of indefinite PP complements, we take the contrast between the articles $a$ and some (1b-4b vs. 8-9) as especially telling. Consider another case where a contrast appears between these two English articles:

a. I consider John a pianist.

b. ??I consider John some pianist.

A prominent approach for analyzing such contrasts between $a$ and some is to assume that the article $a$, as opposed to some, allows a predicative reading for the indefinite. By "predicative" we mean that the indefinite a pianist is interpreted as synonymous with the noun pianist and not as an existential quantifier. In (13a), the application of the predicate denoted by the noun to the entity denoted by John is sanctioned by virtue of the predicative treatment of the $a$ indefinite. ${ }^{4}$

We propose that sentences like we're close tolfar from a gas station in (1) are similarly interpreted using the predicative reading of the indefinite. The spatial expression close tolfar from denotes here a relation between the location of the subject and the location of the predicate gas station. But what does it mean to "locate a predicate"? Intuitively, it is easy to think of the location of entities as their physical location in space or some abstraction thereof. A one-place predicate over physical entities can be thought of as a collection of such entities. We adopt the straightforward assumption that the location of such a collection is the union of the locations of the entities in it. Thus, to be close to (or far from) a gas station, under the predicative reading of this indefinite, is to be close to (far from) the union of the locations of individual gas stations. Now, the existential/universal contrast we have seen follows directly from general assumptions about the meanings of locative expressions. For what is it to be close to or far from an object? It is to be close to (or far from) the closest part of this object. Thus, to be close to the union of gas station locations is to be close to the closest gas station, one gas station nearby is sufficient for this to be true. The location of farther gas stations is irrelevant. Thus, with close to we get an interpretation that is equivalent to the effect of an existential

\footnotetext{
${ }^{4}$ See (Winter 2001) and references therein for more elaborate semantic accounts.
} 
quantifier. To be far from the union of gas station locations is again to be far from the nearest gas station. But as a matter of the meaning of far from, all other gas stations must be further away from that gas station. We therefore get an impression of universal quantification. Thus both sentences in (1) exhibit direct application of a spatial relation to a location of a predicate, and the differences between their interpretations follow from the different meanings of the spatial relations.

This analysis intuitively extends to the other quantificational variability effects we have observed above. In (4a), the dog is inside the union of the locations of the doghouses if it is inside at least one of those regions. By contrast, in (4b), the dog is outside the union of locations if it is outside all of the doghouses. In (5), the usual interpretation of less than 20 m outside $x$ is that the entity is less than $20 \mathrm{~m}$ from $x$ and not inside $x$. If we substitute the combined locations of all doghouses for $x$, we get the quantificational effect in this example: the dog has to be less than $20 \mathrm{~m}$ away from some doghouse and outside all doghouses. The analysis of (6) is similar to that of (5) once we take into account the more complex semantics of above. In (7a), once again we measure distances to the nearest point in a region: an entity $x$ is exactly $100 \mathrm{~m}$ from a region $R$ if there is a point in $R$ which is exactly $100 \mathrm{~m}$ from $x$, and there is no point in $R$ that is closer to $x$. Hence, in (7a) there should be at least one lake which is exactly $100 \mathrm{~m}$ from the house, and no lake can be nearer. The semi-existential/semi-universal effect is thus explained. In (7b) the explanation is similar.

The idea that indefinites can act as the direct arguments of linguistic predicates is not new - it underlies some recent theories of semantic incorporation. A common motivation of these theories, whose origins can be traced back to Carlson's treatment of bare plural indefinites, is to handle exceptional narrow scope interpretations of indefinites. In some cases, incorporation is used in cases where a narrow scope reading is obligatory. For instance, (McNally 1992) uses semantic incorporation to handle obligatory narrow scope with there sentences, as in there isn't a cloud in the sky. Likewise, (Van Geenhoven 1998) uses incorporation to handle obligatory narrow scope in transitive constructions of West Greenlandic, such as John fish-buy-NEG-IND-[tr]-3sg, which means "it is not true that there exists a fish that John buy" and not "there exists a fish that John did not buy". Semantic incorporation is also used by (Zimmermann 1993) and (McNally and Van Geenhoven 2005) to account for cases where indefinites get a narrow scope reading as opposed to other NPs. One of these cases is the well-known behavior of indefinites in intensional contexts. For instance, the sentence Mary is looking for a dog shows an optional, non-existential, de dicto interpretation of the indefinite. This reading is often treated as application of the intensional predicate look for to the indefinite's denotation. In Zimmermann's account this denotation is a property - the intension of a predicative indefinite. In a similar way, we propose that the non-existential reading of constructions like far from a gas station is obtained by application of the relation far from to the predicative denotation of the indefinite. In the next section, we elaborate on the details of this proposal. 


\section{The Formal Semantics of PPs and Indefinite Complements}

So far we have seen that using semantic incorporation of indefinites, the variability in their quantificational force may follow from the different spatial relations that PPs denote. We would now like to analyze in more detail the ways in which spatial relations affect the quantificational behavior of indefinites. In order to do that we need a formal account of the process that was informally described in Section 3. We do that in two steps: first we suggest a semantic structure for PPs with indefinite complements that is based on our analysis of semantic incorporation within locatives. We then use this scheme for characterizing the relations between prepositional meanings and the quantificational interpretation of indefinites in PP complements. It turns out that our incorporation operator expects strong relationships between the semantic (anti-)additivity properties of the prepositional construction and the existential/universal interpretation of the indefinite complement. After studying these relationships, we show some of their interactions the analysis of PP monotonicity, the distribution of negative polarity items and some cases of non-locative prepositions.

\subsection{PP Semantic Structure}

Most theories of prepositions assume that a locative PP establishes a relation between a reference object and a located object. In a sentence like the tree is behind the house, the tree entity is located relative to the object denoted by the PP complement the house. A natural way to represent the meaning of locative prepositions is using regions in a spatial ontology (Nam 1995). A region is a part of the space specified according to the chosen ontology. For the main purposes of this paper it is sufficient to assume that spatial regions are described using sets of points, endowed with the standard set-theoretical operations and relations between them. ${ }^{5}$ Any physical object is mapped to the region it occupies - its eigenspace (Wunderlich 1991). The semantics of locative PPs is given using a prepositional function from regions to sets of regions. A prepositional function receives as argument the eigenspace of the reference object and returns the set of regions possible as eigenspaces for potential located objects. ${ }^{6}$

Consider the following prepositional functions.

\footnotetext{
${ }^{5}$ Ignoring topological relations in the semantics of the PP is an oversimplification, of course, and for the sake of the analysis in Subsection 4.4 of measure phrase modification we will have to introduce distance relations. For more on spatial ontology in the analysis of prepositions see (Nam 1995, Zwarts and Winter 2000, Kracht 2002).

${ }^{6}$ It may sometimes be simpler to define prepositional functions as functions from regions to regions, rather than to sets of regions as it is defined here. In that case the region returned by a prepositional function would have to contain the eigenspace of the located object, rather than have it as a member. This practice is taken in (Zwarts and Winter 2000) but we abandon it here since the present implementation is more useful for introducing our proposal. However, moving back and forth between the two perspectives on prepositions is mechanical, by changing the definition of the "anti-location" function $\operatorname{loc}^{-1}$ (see below).
} 

a. inside $(A)(B) \Leftrightarrow B \subseteq A$
b. outside $(A)(B) \Leftrightarrow B \cap A=\emptyset$
c. close_to $^{\prime}(A)(B) \Leftrightarrow \operatorname{dist}(B, A) \leq c$
d. $\operatorname{far} \operatorname{from}^{\prime}(A) \Leftrightarrow \operatorname{dist}(B, A) \geq f$

The preposition inside denotes here the set containment relation: a sentence like $x$ is inside $y$ is interpreted as stating that the eigenspace of $x$ is contained in the eigenspace of $y$. Similarly, the preposition outside denotes the disjointness relation, and the denotations of the locative constructions close to and far from are defined using a distance function dist in a natural way, with the pragmatic constants $c$ and $f$ as distance values for close and far.

We use the notation 'loc' for the location function that maps any physical entity $x$ to its eigenspace $\operatorname{loc}(x)$. From (Zwarts and Winter 2000) we borrow the idea of using an "inverse" function $l o c^{-1}$, which here receives a set of regions $\mathscr{R}$ and returns the set of objects $l o c^{-1}(\mathscr{R})$ with an eigenspace is $\mathscr{R}$. Formally:

$$
\operatorname{loc}^{-1}(\mathscr{R})=\{x: \operatorname{loc}(x) \in \mathscr{R}\}
$$

The semantic structure that we assume for locative PPs is summarized in (16).

$$
\operatorname{loc}^{-1}(P(\operatorname{loc}(x)))
$$

- $x$ is the reference object of the PP - a physical entity of type $e$.

- loc is the location function - from $e$-type entities to their eigenspace regions.

- $P$ is the prepositional function of the PP - a function from regions to sets of regions, or equivalently - a relation between regions.

- $l o c^{-1}$ is the anti-location function - from a set of regions $\mathscr{R}$ to the set of entities whose eigenspace is in $\mathscr{R}$.

For example, in a locative PP like outside the meadow, the loc function maps the meadow entity $\mathbf{m}^{\prime}$ to its eigenspace $M=\operatorname{loc}\left(\mathbf{m}^{\prime}\right)$. The locative outside maps the region $M$ to the set of regions that do not overlap $M$. The $l o c^{-1}$ function returns the set of entities that occupy these regions - the one-place predicate that specifies the entities outside the meadow. As a result, the sentence the house is outside the meadow receives the following semantic analysis:

$$
\begin{aligned}
& \left(\operatorname{loc}{ }^{-1}\left(\text { outside }^{\prime}\left(\operatorname{loc}\left(\mathbf{m}^{\prime}\right)\right)\right)\right)\left(\mathbf{h}^{\prime}\right) \\
& \Leftrightarrow \operatorname{loc}\left(\mathbf{h}^{\prime}\right) \in \text { outside }^{\prime}\left(\operatorname{loc}\left(\mathbf{m}^{\prime}\right)\right) \\
& \Leftrightarrow H \in \text { outside }^{\prime}(M) \\
& \Leftrightarrow H \cap M=\emptyset
\end{aligned}
$$

In words: the eigenspaces $H$ and $M$ of the house and the meadow are disjoint.

The semantic structure in (16), which is adopted with some modifications from Zwarts and Winter's work, is used here as the basis for our treatment of indefinite PP complements. To implement our assumption that a prepositional function 
can receive a predicate as its direct argument, we apply an incorporation operator. Using this operator, instead of applying the prepositional function $P$ to the eigenspace of a single entity as in (16), we apply it to the union of eigenspaces of entities in a predicate's extension. To get this union, we define the location function $l o c \cup$ over predicates as follows.

$$
\operatorname{loc}^{\cup}(F)=\bigcup\{\operatorname{loc}(x): F(x)\}
$$

Here $F$ is the denotation of a predicate over physical entities. The resulting semantic structure of a PP with a predicative complement is the following:

$$
\operatorname{loc}^{-1}\left(P\left(\operatorname{loc}^{\cup}(F)\right)\right)
$$

- $F$ is a predicate of type $e t$ over reference objects.

- $l o c^{\cup}$ is the location function over predicates - from et-type predicates to regions.

- $\quad P$ and $l o c^{-1}$ are as described in (16).

For example, in the expression outside a meadow, the semantic structure we assume is the following:

$$
\operatorname{loc}^{-1}\left(\operatorname{outside}^{\prime}\left(\operatorname{loc}^{\cup}\left(\operatorname{meadow}_{e t}^{\prime}\right)\right)\right)
$$

The function $l o c \cup$ sends the predicative indefinite a meadow to the union of the

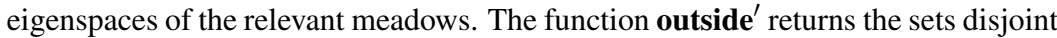
to that union, and $l o c^{-1}$ returns the entities that occupy such sets: the entities that do not overlap with any of the meadows. In formula, the sentence the house is outside a meadow (cf. 4b) is analyzed as follows.

(21) The house is outside a meadow.

$$
\begin{aligned}
& \left(\operatorname{loc}^{-1}\left(\text { outside }^{\prime}\left(\operatorname{loc} \cup\left(\text { meadow }^{\prime}{ }\right)\right)\right)\right)\left(\mathbf{h}^{\prime}\right) \\
& \Leftrightarrow \operatorname{loc}\left(\mathbf{h}^{\prime}\right) \in \operatorname{outside}^{\prime}\left(\bigcup\left\{\operatorname{loc}(x): \text { meadow }^{\prime}(x)\right\}\right) \\
& \Leftrightarrow H \in\left\{R: R \cap \bigcup\left\{\operatorname{loc}(x): \text { meadow }^{\prime}(x)\right\}=\emptyset\right\} \\
& \Leftrightarrow H \cap \bigcup\left\{\operatorname{loc}(x): \operatorname{meadow}^{\prime}(x)\right\}=\emptyset
\end{aligned}
$$

Assuming that the set of meadows is finite, we get:

$$
\begin{aligned}
& \Leftrightarrow \forall x\left[\operatorname{meadow}^{\prime}(x) \rightarrow H \cap \operatorname{loc}(x)=\emptyset\right] \\
& \Leftrightarrow \forall x\left[\operatorname{meadow}^{\prime}(x) \rightarrow\left(\operatorname{loc}^{-1}\left(\operatorname{outside}^{\prime}(\operatorname{loc}(x))\right)\right)\left(\mathbf{h}^{\prime}\right)\right]
\end{aligned}
$$

In words: for every meadow $x$, the house is outside $x$.

In this derivation we see how our incorporation-based analysis using the $l o c^{\cup}$ function derives a pseudo-universal interpretation of the predicative indefinite with the preposition outside. Broadening our view, we would like to have a general characterization of the quantificational effects with predicative indefinites as dependent on the prepositional function they complement. We now move on to introduce our analysis of these semantic dependencies. 


\subsection{Anti-Additivity and Pseudo-Universal Indefinites}

The universal analysis of the locative outside a meadow in (21) relies on a special property of the locative outside. A house that is outside a finite union of eigenspaces is outside each of these eigenspaces, and vice versa. Our treatment of the prepositional function for outside as the disjointness relation naturally supports this property. Generalizing, we say that the preposition outside is anti-additive where antiadditivity is defined below, following (Zwarts 1998, Sánchez-Valencia et al. 1994, Nam 1997). ${ }^{7}$

Definition 1 A preposition function $P$ is called anti-additive if for all regions $A, B$ :

$$
P(A \cup B)=P(A) \cap P(B) .
$$

In the semantic structure we have assumed, we get the universal interpretation for any anti-additive prepositional function $P$ due to the following equivalence for every predicate $F$ that holds of a finite set of entities: ${ }^{8}$

$$
\begin{aligned}
y & \in \operatorname{loc}^{-1}\left(P\left(\operatorname{loc} \cup\left(F_{e t}\right)\right)\right) & & \\
& \Leftrightarrow \operatorname{loc}(y) \in P(\bigcup\{\operatorname{loc}(x): F(x)\}) & & \text { (by definition of } \left.l o c^{-1} \text { and } l o c^{\cup}\right) \\
& \Leftrightarrow \operatorname{loc}(y) \in \bigcap_{x: F(x)} P(\operatorname{loc}(x)) & & \text { (definition of anti-additivity) } \\
& \Leftrightarrow \forall x[F(x) \rightarrow \operatorname{loc}(y) \in P(\operatorname{loc}(x))] & & \\
& \Leftrightarrow \forall x\left[F(x) \rightarrow y \in \operatorname{loc}^{-1}(P(\operatorname{loc}(x)))\right] & & \text { (by definition of } \left.l o c^{-1}\right)
\end{aligned}
$$

Now it is clear why the locatives outside and far from trigger universal readings of predicative indefinite complements. These prepositions denote anti-additive functions, as informally illustrated by the following equivalences.

a. We're outside Eurasia $\Leftrightarrow$ we're outside Europe and outside Asia.

b. We're far from Eurasia $\Leftrightarrow$ we're far from Europe and far from Asia.

The anti-additivity of the prepositional functions that we assumed in (14) for outside and far from formally reflects their inferential behavior in (23). This anti-additivity does not appear with the other prepositional constructions that we have considered. Especially, the locatives inside and close to are not anti-additive, as the following invalid entailments show.

a. We're inside Eurasia $\nRightarrow$ We're inside Europe.

We're inside Eurasia $\nRightarrow$ We're inside Asia.

b. We're close to Eurasia $\nRightarrow$ We're close to Europe.

We're close to Eurasia $\nRightarrow$ We're close to Asia.

\footnotetext{
${ }^{7}$ In a principle referred to as the principal filter property, (Winter 2001) takes anti-additivity to be responsible for the union-like ("collective") interpretation of intersections of generalized quantifiers.

${ }^{8}$ The behavior of prepositions over infinite unions is a question we set aside here, like many other mathematical questions about the behavior of prepositional functions.
} 
The lack of anti-additivity with inside and close to accounts for the lack of "universal" interpretation for indefinites with those prepositional constructions, as antiadditivity is necessary for the equivalence in (22) to hold.

Anti-additivity is closely related to the notion of downward monotonicity. Standardly, a function is called upward (downward) monotone if it preserves (reverses) order on its arguments. Zwarts and Winter (2000) demonstrate that like other expressions in natural language, locative prepositions exhibit a variety of monotonicity properties. ${ }^{9}$ The following definition standardly describes the monotonicity of prepositional functions. ${ }^{10}$

\section{Definition 2 Let $P$ be a prepositional function.}

1. $P$ is upward monotone if for all regions $A, B: A \subseteq B \Rightarrow P(A) \subseteq P(B)$.

2. $P$ is downward monotone iffor all regions $A, B: A \subseteq B \Rightarrow P(B) \subseteq P(A)$.

Downward monotonicity follows directly from anti-additivity. Intuitively, the leftto-right entailments in (23) establish the downward monotonicity of the prepositional constructions outside and far from. By contrast, the non-anti-additive prepositional constructions inside and close to are furthermore not downward monotone, as the invalid entailments in (24) illustrate.

The examples we know for downward monotone prepositional constructions are outside and far from, as well as the semantically similar construction at least/more than $n$ meters from. It seems little coincidence that these constructions are furthermore anti-additive. Most prepositional constructions we know have a property that we can call cumulativity. A prepositional function $P$ is cumulative if for all regions $A, B: P(A \cup B) \supseteq P(A) \cap P(B)$. Anti-additivity is equivalent to cumulativity plus downward monotonicity. Cumulativity is prevalent with prepositional constructions, also with those that are not downward monotone. Consider for instance the following examples, with the prepositions above and close to.

a. The cloud is above the house and above its grounds $\Rightarrow$ the cloud is above the premises.

b. We're close to Europe and close to Asia $\Rightarrow$ we're close to Eurasia.

However, cumulativity is not a universal property of prepositional constructions. Consider for instance a satellite over Canada, which is diagonally above Alaska, as well as diagonally above the bulk of the US. Even ignoring Hawaii, it would be strange to assert that the satellite is diagonally above US territory. Thus, diagonally above is unlikely to be a cumulative prepositional construction. This is more graphically illustrated in Figure 2a.

\footnotetext{
${ }^{9}$ See (Zimmermann 2006) for a recent study of the monotonicity properties of intensional verbs, which as we mentioned above, are highly related to the theory of semantic incorporation.

${ }^{10} \mathrm{Zwarts}$ and Winter refer to point monotonicity since in their semantics, the region argument of a prepositional function is a set of points. Simplifying, we here use the term monotonicity. It should be noted that the modification of the type for prepositional functions that we introduce here leads to a different characterization of these functions in terms of their monotonicity. Empirical matters do not hinge on this fact too much, since we have adjusted here the operation of the $l o c^{-1}$ function to match the different type we assume for prepositions.
} 


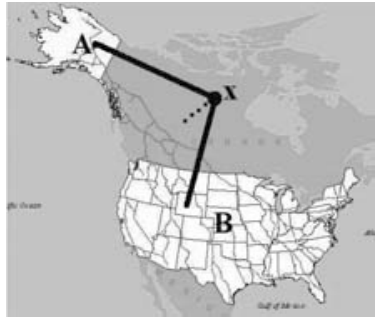

(a)

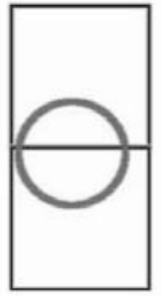

(b)

Figure 2: (a) $x$ is diagonally above $A$ and diagonally above $B$, but not diagonally above $A \cup B$. (b) The circle is in the squares but not in a square.

\subsection{Additivity and Pseudo-Existential Indefinites}

Preposition additivity is defined similarly to anti-additivity:

Definition 3 A preposition function $P$ is called additive if for all regions $A, B$ :

$$
P(A \cup B)=P(A) \cup P(B) .
$$

Unlike the clear parallelism between universal interpretations of indefinites and the anti-additivity of far from and outside, the relations between additivity and existential interpretations require a more complex semantic analysis. Consider first the prepositional construction close to and the empirical status of the following additivity entailment tests.

$$
\begin{aligned}
& \text { a. We're close to Eurasia } \Rightarrow \text { We're close to Europe or close to Asia. } \\
& \text { b. We're close to Eurasia } \stackrel{?}{\models} \text { We're close to Europe or close to Asia. }
\end{aligned}
$$

There is little question about the validity of the entailment in (26a). At first blush, also the entailment in (26b) seems perfectly valid. This entailment is characteristic of the upward monotone prepositional constructions. But does it hold for close to? Suppose that we are in Asia and have gotten close to European territories. Thus the first disjunct in the righthand side of (26b) is certainly true. Does it follow that we are close to Eurasia in such a case? To be sure, it would be rather infelicitous to state that we are close to Eurasia when in fact, we are in Eurasia. Now we have one of two theoretical alternatives: either (i) conclude that close to is not an upward monotone preposition; or (ii) assume that close to is upward monotone, and attribute the infelicity of $A$ is close to $B$ when $A$ is in $B$ to a pragmatic effect. Fortunately, we do not have to choose between these options here since both of them are consistent with our account of indefinite PP complements. Under the non-monotonicity assumption (i), a sentence like we're close to a gas station is simply non-existential, since it requires, in addition to the existence of a gas station nearby, that we are outside all gas stations. Thus, non-additivity in this case would 
correspond to its non-existentiality. Vice versa: if we adopt the pragmatic line in (ii), then close to is assumed to be additive, and our semantic analysis of the indefinite in close to a gas station would remain purely existential. The additional implication that we're outside all gas stations would then be analyzed as pragmatic.

The situation with the preposition inside is more complicated. While being upward monotone (cf. $27 \mathrm{~b}$ below), this preposition is clearly non-additive, as the invalid entailment (27a) shows.

a. This territory is inside Eurasia $\nRightarrow$ This territory is inside Europe or inside Asia.

b. This territory is inside Eurasia $\Leftarrow$ This territory is inside Europe or inside Asia.

From the formal proposal above, we would therefore expect the indefinite in a sentence like (28) below to be non-existential.

(28) The circle is inside a square.

For instance, a circle that is contained in two squares as in Figure $2 b$ would be expected to satisfy a non-existential reading of (28). But evidently, this sentence is univocally false in Figure $2 \mathrm{~b}$, and the indefinite in it is purely existential. Note that this is no longer the case if we replace the singular a square in (28) by a plural like (the) squares: the sentences the circle is inside (the) squares are true in Figure $2 \mathrm{~b}$. We thus hypothesize that the existential reading of (28) results from some special aspects of the meaning of the singular indefinite, and not simply from the meaning of the preposition. That non-existential singular indefinites behave differently than plurals has been observed in the literature on genericity. For instance, consider the contrast in collective generic sentences like tigers $/ *$ a tiger gather $(s)$ at night. We speculate that the existential reading of (28) may be a result of this "singularproperty" aspect of the meaning of singular indefinites. However, we leave the investigation of this aspect to further research.

\subsection{MP Monotonicity and Prepositional (Anti-)Additivity}

Reconsider the sentences in (2), restated below.
a. The house is $\left\{\begin{array}{l}\text { at most } \\ \text { less than }\end{array}\right\} 2 \mathrm{~km}$ from a lake.
b. The house is $\left\{\begin{array}{l}\text { at least } \\ \text { more than }\end{array}\right\} 2 \mathrm{~km}$ from a lake.

In these sentences we have seen a correlation between the identity of the modified numeral within the measure phrase and the existential/universal interpretation of the indefinite. Abstracting away from the measuring unit (meters, kilometers, etc.), let us assume that measure phrases denote subsets of the non-negative real numbers $\mathbf{R}^{+}{ }^{11}$ Generalizing the definitions of the denotations in (14c)-(14d) of the prepo-

\footnotetext{
${ }^{11}$ Measuring units will only require multiplying these numbers by the proper constants, e.g. 0.001 for meters and 1 for kilometers, or any other constants with the proper relation between them (e.g. 1
} 
sitional constructions close to and far from, let us adopt the following definition for the denotation of the construction MP from, where $\mathbf{M P} \mathbf{P}^{\prime}$ is the denotation of the measure phrase (e.g. less/more than $2 \mathrm{~km}$ ).

$$
\text { MP_from }{ }^{\prime}(A)(B) \Leftrightarrow \operatorname{dist}(B, A) \in \mathbf{M P}^{\prime}
$$

We assume the following denotations for the different MPs in (29):

$$
\begin{aligned}
& \text { a. at_most_2 } \mathbf{k m}^{\prime}=\{r: r \leq 2\} \\
& \text { less_than_2(km' }=\{r: r<2\} \\
& \text { b. at } \_ \text {least } \_\mathbf{k m}^{\prime}=\{r: r \geq 2\} \\
& \text { more_than } 2 \mathbf{k m}^{\prime}=\{r: r>2\}
\end{aligned}
$$

According to these denotations and the definition in (30), we get the following denotations for the prepositional constructions in (29).

a. at_most_2 $\mathbf{k m}$ from $(A)(B) \Leftrightarrow \operatorname{dist}(B, A) \leq 2$

less_than_2km_from $(A)(B) \Leftrightarrow \operatorname{dist}(B, A)<2$

b. at_least $2 \mathbf{k m} \_$from $(A)(B) \Leftrightarrow \operatorname{dist}(B, A) \geq 2$ more_than_2km_from $(A)(B) \Leftrightarrow \operatorname{dist}(B, A)>2$

Trivially, the MP denotations in (31a) and (31b) are downward/upward monotone respectively in the set $\mathbf{R}^{+}$of non-negative real numbers. ${ }^{12}$ What is a little bit surprising is that we have already seen that the denotations of the prepositional correlates of these MPs - in (32a) and (32b) respectively - may have the opposite (upward/downward) monotonicity. ${ }^{13}$ Furthermore, as argued above, these prepositional constructs are additive and anti-additive, respectively. How does monotonicity reverse when composing MPs into prepositional constructions? The answer is not too hard when looking further into the behavior of the distance relation dist in definition (30).

Following Zwarts and Winter (2000), we define the distance function using a metric on the points in our ontology. ${ }^{14}$ Given such a metric $d$, we can define distance between regions as the following infimum: ${ }^{15}$

For any two regions $A, B: \operatorname{dist}(A, B)=\inf \{d(x, y): x \in A, y \in B\}$.

For this definition, we show the following fact about the (anti-)additivity of the denotation (30) of a prepositional expression $M P$ from, as a function of the monotonicity of the measure phrase $M P$.

\footnotetext{
for meters and 1000 for kilometers).

${ }^{12}$ For a detailed analysis of the semantics of MPs and their MP modification constructions see Winter (2005).

${ }^{13}$ For the sake of the discussion here we assume that at most/less than $2 \mathrm{~km}$ from are indeed upward monotone, as the pragmatic assumption on the compatibility of close to with inside would expect.

${ }^{14}$ For a set $X$, a metric over $X$ is a function from $X \times X$ to $\mathbf{R}^{+}$that satisfies for all $x, y, z \in X$ : $d(x, y)=d(y, x), d(x, y)+d(y, z) \geq d(x, z)$, and $d(x, y)=0$ iff $x=y$.

${ }^{15}$ Recall that infimum is the greatest lower bound for (possibly) infinite sets - the generalization of the minimum function for finite sets.
} 
Fact 1 Let $\mathbf{M P}^{\prime}$ be a set $M \subseteq \mathbf{R}^{+}$of non-negative reals, and let $f_{M}=\mathbf{M P}$ from'.

If $M$ is upward monotone in $\mathbf{R}^{+}$then $f_{M}$ is anti-additive.

If $M$ is downward monotone in $\mathbf{R}^{+}$then $f_{M}$ is additive.

Intuitively, when an MP is upward monotone, the distance between $A \cup B$ and $C$ is in the MP's denotation if and only if both distances $\operatorname{dist}(A, C)$ and $\operatorname{dist}(B, C)$ are in the MP's denotation. Similarly, when an MP is downward monotone, the distance between $A \cup B$ and $C$ is in the MP's denotation if and only if at least one of the distances $\operatorname{dist}(A, C)$ and $\operatorname{dist}(B, C)$ is in the MP's denotation. This property stems from the property $\operatorname{dist}(A \cup B, C)=\min (\operatorname{dist}(A, C)$, $\operatorname{dist}(B, C))$, which is a direct result of the definition of the distance function as an infimum.

Not every MP is upward monotone or downward monotone. Some MPs, such as exactly $10 \mathrm{~km}$ or between $10 \mathrm{~m}$ and $20 \mathrm{~m}$ are neither upward nor downward monotone. However, these MPs denote conjunctions of monotonic functions. As a result, the resulting prepositional constructions exactly $10 \mathrm{~km}$ from and between $10 \mathrm{~m}$ and $20 \mathrm{~m}$ from denote intersections of an anti-additive prepositional function with an additive prepositional function. This accounts for the combined existentialuniversal effects with such MPs that we observed in Section 2.

\subsection{Further Implications: Negative Polarity Items and Non-Locative Prepositions}

The characterization of some locatives as anti-additive (or downward monotone) functions has ramifications for the analysis of negative polarity items (NPIs). In the literature on NPIs since (Ladusaw 1979) it has become customary to observe that downward monotone operators license the appearance of NPIs like any or ever in their scope, whereas upward monotone and non-monotone operators do not. It is therefore unsurprising that we observe the following contrasts between locative prepositions with respect to their NPI licensing.

a. We're far from/?close to any gas station.

b. The house is more than $2 \mathrm{~km} /$ ?less than $2 \mathrm{~km}$ from any lake.

c. This factory is outside/?inside any industrial area.

The distinction between anti-additive functions and other downward monotone functions was used in the literature on NPIs (Zwarts 1998, Sánchez-Valencia et al. 1994, Nam 1997). The so-called strong NPIs like English yet and Dutch ook maar were claimed to require anti-additive contexts, whereas weak NPIs like English any or ever were claimed to only require downward monotone environments for their licensing. We will leave the characterization of NPIs with locative prepositions to further research.

Although as far as we know, locative prepositions were not extensively studied in the literature on NPIs, temporal prepositions were. Consider the following English examples from (Nam 1997):

a. Mary arrived before anyone left the party.

b. *Mary arrived after anyone left the party. 
Based on the analysis of temporal before in Landman (1991), Sánchez-Valencia et al. (1994) argue that before is an anti-additive preposition. (Nam 1997) strengthens this claim by studying strong NPIs in Korean and Japanese. Nam points out that NPIs like teisang (Korean, 'anymore') and soreizyoo (Japanese, 'anymore') appear in complements of before although they do not appear in downward monotone environments that are not anti-additive.

Given this background, it is surprising that it is not easy to attest a difference between before and after when they appear with indefinite complements. For instance, both sentences below have an existential interpretation for the indefinite description a war.

a. This shelter was built before a war.

b. This shelter was built after a war.

We would expect sentence (35a) to have a universal interpretation equivalent to this shelter was built before all wars. This expectation is not borne out, for reasons that we will not study here. However, we should point out that contrasts that are very similar to the ones we observed in (6), repeated below, can also be observed with temporal PPs.

(36) The bird is more than $20 \mathrm{~m}$ above a cloud. (=6)

In (36), the bird is claimed to be more than $20 \mathrm{~m}$ above all the clouds below it. Similarly, in (37) below, it is claimed that the shelter was built more than two years after/before all wars that started before/after it was built.

(37) This shelter was built more than two years after/before a war.

We conclude that incorporation is also useful for the analysis of temporal PPs with indefinite complements, but its exact interaction with the meanings of temporal prepositions requires further study. Another issue that we will have to leave for further research is the interaction of predicative indefinites with directional prepositions. ${ }^{16}$ Consider the following example.

(38) We went around a lake.

Sentence (38) can only be interpreted using existential quantification over lakes. There is no interpretation of (38) according to which we went around a collection of lakes. We believe that this apparent lack of incorporation effects is similar to the effect we mentioned above with the singular indefinite and the locative inside in sentence (28).

\section{Conclusions}

As pointed out in the introduction, we believe that PP complements should be put alongside generics and donkey anaphora as one of the apparently extensional contexts that support non-existential interpretations of indefinites. As in other similar

\footnotetext{
${ }^{16}$ Thanks to Joost Zwarts for pointing out this problem to us.
} 
cases, our goal in this paper was to explain non-existential effects as an epiphenomenon of the semantics of the indefinite's environment. In the case of spatial locative prepositions, we aimed to show that non-existential effects with indefinites result from direct predication of the prepositional meaning to the predicate denoted by the indefinite, combined with the denotational properties of spatial PPs. The use of semantic incorporation has become popular in recent years for treating intensional transitive constructions as well as other phenomena in various languages. While these applications of incorporation are certainly new, we believe that they ultimately reshape and extend Carlson's classical treatment of generic bare plurals as kind referring. In the areas of intensionality and genericity, there is still an ongoing debate whether semantic incorporation is advantageous to quantificational techniques for treating indefinites. Many authors have argued that genericity operators and intensional existential quantifiers are advantageous to kinds and properties in the treatment of genericity and intensionality. In relation to spatial locative PPs, however, we have shown strong evidence for the predicative analysis of indefinites as the direct argument of prepositional relations. On the whole we believe that these evidence support non-quantificational approaches to indefinites, and that their broader theoretical ramifications merit further research.

\section{References}

Carlson, Gregory. N.: 1977, Reference to Kinds in English, Doctoral Dissertation, UMass.

Heim, Irene: 1982, The Semantics of Definite and Indefinite Noun Phrases, Doctoral Dissertation, UMass.

Kamp, Hans: 1981, 'A theory of truth and semantic representation', in J. Groenendijk et al (eds.), Formal Methods in the Study of Language. Mathematisch Centrum, Amsterdam.

Kracht, Marcus: 2002, 'On the semantics of locatives', Linguistics and Philosophy 25, 157-232.

Ladusaw, William A.: 1979, Polarity Sensitivity as Inherent Scope Relations, Doctoral Dissertation, University of Texas at Austin.

Landman, Fred: 1991, Structures for Semantics. Kluwer Academic Publishers, Dordrecht.

McNally, Louise and Van Geenhoven, Veerle: 2005, 'On the property analysis of opaque complements', Lingua 115, 885-914.

McNally, Louise: 1992, An Interpretation for the English Existential Construction, Doctoral Dissertation, UCSC.

Nam, Seungho: 1995, The Semantics of Locative PPs in English, Doctoral Dissertation, UCLA.

Nam, Seungho: 1997, 'The semantics of before and negative polarity', paper presented at Japanese-Korean linguistic conference. Cornell University.

Sánchez-Valencia, Valencia; van der Wouden, Ton and Zwarts, Frans: 1994, 'Po- 
larity, veridicality and temporal connectives', in Proceedings of the 9th Amsterdam Colloquium.

Schwarzschild, Roger: 2002, 'Singleton indefinites', Journal of Semantics 19, 289314.

Van Geenhoven, Veerle: 1998, Semantic Incorporation and Indefinite Descriptions. CSLI.

Winter, Yoad: 2001, Flexibility Principles in Boolean Semantics: coordination, plurality and scope in natural language. MIT Press, Cambridge, Massachusetts.

Winter, Yoad: 2005, 'Cross-Categorial Restrictions on Measure Phrase Modification', Linguistics and Philosophy 28, 233-267.

Wunderlich, Dieter: 1991, 'How do prepositional phrases fit into compositional syntax and semantics?', Linguistics 29, 591-621.

Zimmermann, Thomas Ede: 2006, 'Monotonicity in Opaque Verbs', Linguistics and Philosophy 29, 715-761.

Zimmermann, Thomas Ede: 1993, 'On the Proper Treatment of Opacity in Certain Verbs', Natural Language Semantics 1, 149-179.

Zwarts, Frans: 1998, 'Three types of polarity', in F. Hamm and E. Hinrichs (eds.), Plurality and quantification, 177-238. Dordrecht: Kluwer.

Zwarts, Joost and Winter, Yoad: 2000, 'Vector Space Semantics: a modeltheoretic analysis of locative prepositions', Journal of Logic, Language and Information 9, 171-213. 